\title{
P7-21 Troubles sensoriels et désafférentation
}

\author{
C) Springer-Verlag France 2012
}

\author{
P7-21.72 \\ Cécité corticale et leucoencéphalopathie \\ postérieure régressive \\ S.-S. Ait ${ }^{1}$, T. Gilbert, Pr Bonnefoy \\ CHLS, Pierre-Bénite, France
}

Introduction : La leucoencéphalopathie postérieure régressive(LEPR) est un syndrome clinicoradiologique associant une symptomatologie neurologique comportant des céphalées, un syndrome confusionnel, des crises d'épilepsie et des troubles visuels allant de l'hémianopsie à la cécité corticale. Le diagnostic est évoqué à l'aide d'une IRM cérébrale. Un diagnostic précoce permettant une prise en charge adaptée du facteur déclenchant conduit à une régression complète de la symptomatologie. Nous rapportons un cas de crise convulsive associée à une cécité corticale ayant totalement régressé en une semaine chez une patiente de 86 ans.

Objectifs : Une femme de 86 ans a été hospitalisée à la suite d'une chute. À son admission, la patiente était confuse et présentait des clonies du membre supérieur droit et une cécité bilatérale. Dans ses antécédents, on notait un diabète non insulinodépendant, une hypertension artérielle. L'examen clinique retrouvait une cécité corticale. L'évolution a été favorable en une dizaine de jours, le syndrome confusionnel ayant régressé avec récupération de l'acuité visuelle. L'IRM objectivait en séquence T2 un hypersignal cortical et cortico-sous-cortical bilatéral intéressant les lobes occipitaux et pariétaux associé à un hypersignal minime sur la séquence de diffusion. L'ensemble des éléments cliniques et radiologiques était évocateur d'une encéphalopathie postérieure régressive d'origine hypertensive probable.

Méthodes : La LEPR a été décrite la première fois en 1996 par Hinchey et al. Il s'agit d'un syndrome clinicoradiologique associant des manifestations neurologiques telles que des céphalées, des syndromes confusionnels, des troubles de la vigilance à des troubles visuels comme un scotome, une hémianopsie ou une cécité corticale, des crises d'épilepsie partielles ayant tendance à se généraliser associées à des signes neuroradiologiques. L'IRM est l'examen de référence, retrouvant dans les zones postérieures de façon bilatérale des hypersignaux sur les séquences T2 et FLAIR et des lésions en iso- ou hyposignal en $\mathrm{T} 1$ de la substance blanche, la séquence de diffusion et le coefficient apparent de diffusion permettent de différencier les lésions vasogéniques des lésions ischémiques irréversibles. Plusieurs mécanismes physiologiques sont suspectés dont une défaillance de l'autorégulation de la vascularisation cérébrale par un dysfonctionnement des cellules endothéliales de la barrière hématoencéphalique entraînant un œdème vasogénique. Les circonstances favorisantes de LEPR sont nombreuses. La cause la plus fréquemment retrouvée est une élévation brutale de la tension artérielle. Les autres affections habituellement associées sont l'éclampsie, l'insuffisance rénale, le purpura thrombocytopénique, les maladies de système dont les connectivites et vasculites, maladies auto-immunes, les infections virales, les troubles électrolytiques, les immunosuppresseurs (cyclosporine et tacrolimus), les corticoïdes à fortes doses, les transfusions sanguines, certaines chimiothérapies. Le traitement consiste en une équilibration de la TA et l'interruption des médicaments en cause. Dès lors, les manifestations neurologiques régressent dans un délai moyen d'une à deux semaines.

Conclusion : La LEPR est un syndrome potentiellement grave de par ses complications cérébrales ischémiques ou hémorragiques. Un diagnostic et une prise en charge précoces permettent d'améliorer le pronostic.

Conflit d'intérêt : les auteurs déclarent ne pas avoir de conflit d'intérêt. 